\title{
Frequencies of wave packets of whistler-mode chorus inside its source region: a case study
}

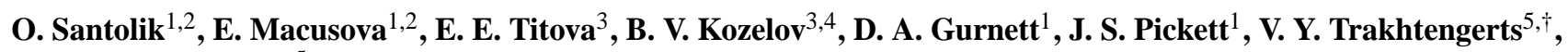 \\ and A. G. Demekhov ${ }^{5}$ \\ ${ }^{1}$ University of Iowa, Iowa City, Iowa, USA \\ ${ }^{2}$ permanently at Institute of Atmospheric Physics CAS, Prague and Charles University, Faculty of Mathematics and Physics, \\ Prague, Czech Republic \\ ${ }^{3}$ Polar Geophysical Institute, Apatity, Russia \\ ${ }^{4}$ Department of Physics and Technology, University of Troms $\varnothing$, Troms $\varnothing$, Norway \\ ${ }^{5}$ Institute of Applied Physics, Nizhny Novgorod, Russia \\ $\dagger$ deceased
}

Received: 23 January 2008 - Accepted: 18 April 2008 - Published: 11 June 2008

\begin{abstract}
Whistler-mode chorus is a structured wave emission observed in the Earth's magnetosphere in a frequency range from a few hundreds of $\mathrm{Hz}$ to several $\mathrm{kHz}$. We investigate wave packets of chorus using high-resolution measurements recorded by the WBD instrument on board the four Cluster spacecraft. A night-side chorus event observed during geomagnetically disturbed conditions is analyzed. We identify lower and upper frequencies for a large number of individual chorus wave packets inside the chorus source region. We investigate how these observations are related to the central position of the chorus source which has been previously estimated from the Poynting flux measurements. We observe typical frequency bandwidths of chorus of approximately $10 \%$ of the local electron cyclotron frequency. Observed time scales are around $0.1 \mathrm{~s}$ for the individual wave packets. Our results indicate a lower occurrence probability for lower frequencies in the vicinity of the central position of the source compared to measurements recorded closer to the outer boundaries of the source. This is in agreement with recent research based on the backward wave oscillator theory.
\end{abstract}

Keywords. Magnetospheric physics (Plasma waves and instabilities) - Radio science (Magnetospheric physics) Space plasma physics (Waves and instabilities)

\section{Introduction}

Whistler-mode chorus is generated by a nonlinear mechanism involving wave-particle interactions with energetic

Correspondence to: O. Santolik

(ondrej.santolik@mff.cuni.cz) electrons (Helliwell, 1967; Tsurutani and Smith, 1974; Nunn et al., 1997; Trakhtengerts, 1999). Close to its source region in the vicinity of the geomagnetic equator (LeDocq et al., 1998; Parrot et al., 2003; Santolík et al., 2004, 2005), chorus is often divided into two frequency bands. They are separated by a gap at about one half of electron cyclotron frequency. Chorus typically has a discrete structure of separate wave packets (elements) in a frequency range from a few hundreds of $\mathrm{Hz}$ to several $\mathrm{kHz}$ (see reviews by Omura et al., 1991; Sazhin and Hayakawa, 1992, and references therein).

Trakhtengerts et al. (2007) showed that the spectrum of chorus wave packets lacks lower frequencies close to the center of the source region and explained this effect on the basis of the backward wave oscillator model (Trakhtengerts, 1995, 1999; Trakhtengerts and Rycroft, 2000; Trakhtengerts et al., 2004). This model links the frequency of chorus wave packets to the parallel component of the velocity of a steplike deformation of the electron distribution function. This velocity decreases as the resonant electrons move along the magnetic field lines through the chorus source region. Different wave frequencies are therefore generated at different positions within the source. For chorus wave packets which rise in frequency, lowest frequencies are generated closer to the boundaries of the source region. Thus, only the upperfrequency parts of chorus wave packets are visible well inside this region.

Similarly to Trakhtengerts et al. (2007), we investigate these wave packets from detailed frequency-time power spectrograms calculated using waveforms measured by the WBD instrument (Gurnett et al., 1997, 2001) on board the four Cluster spacecraft. We use the same data set recorded on 18 April 2002 during a geomagnetic storm when the $D_{s t}$ index decreased below $-120 \mathrm{nT}$, the $K_{p}$ index reached $7^{\circ}$, and

Published by Copernicus Publications on behalf of the European Geosciences Union. 


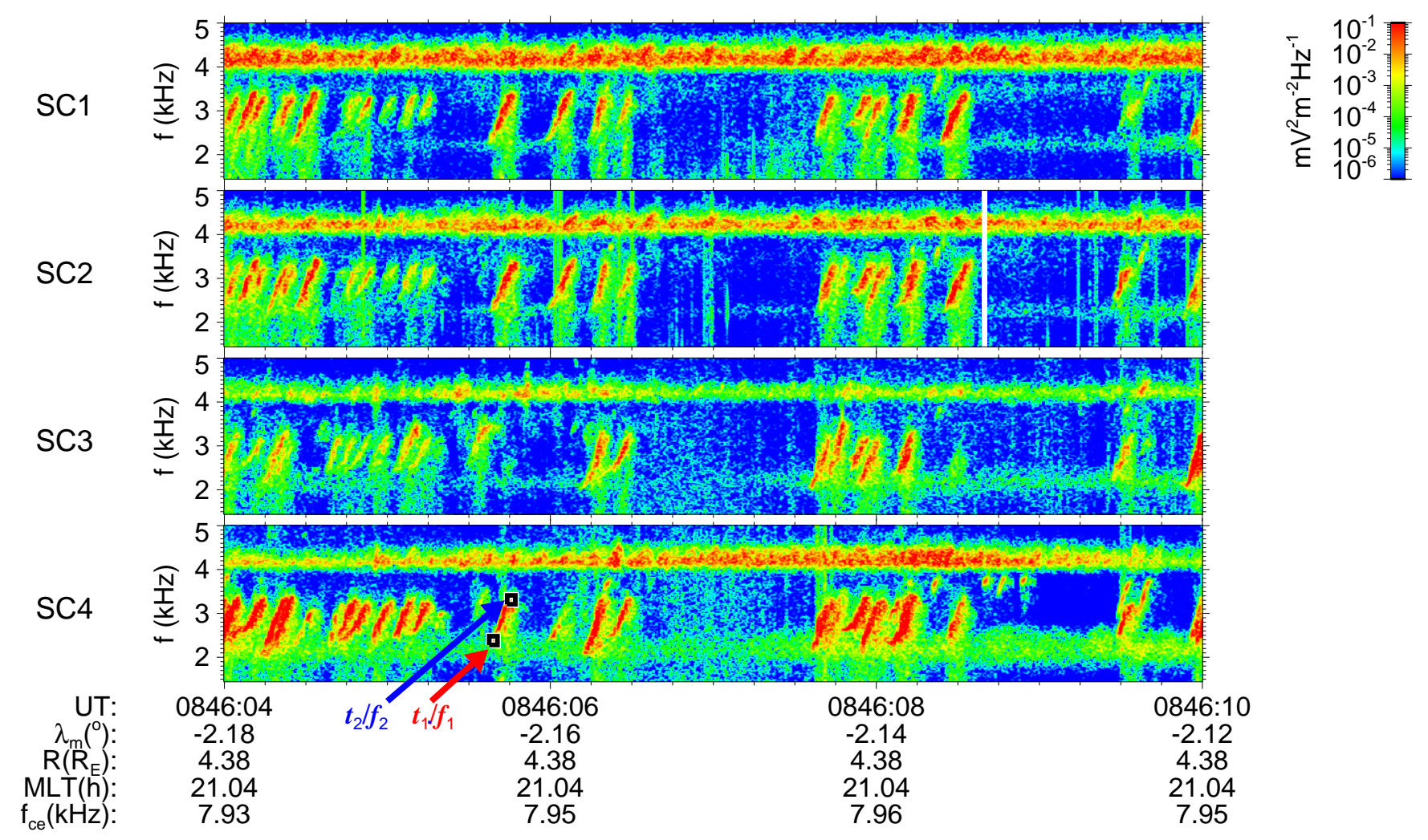

Fig. 1. Example frequency-time spectrogram of the power-spectral density of electric field fluctuations measured by the WBD instrument on board the four Cluster spacecraft (SC1-SC4) on 18 April 2002. Magnetic latitude, radial distance, magnetic local time, and the local electron cyclotron frequency from averaged data of the FGM instrument (Balogh et al., 2001) are given on the bottom for Cluster 4. Arrows illustrate an example of the starting point $\left[t_{1}, f_{1}\right]$ (red) and the final point $\left[t_{2}, f_{2}\right]$ (blue) of a single chorus wave packet.

the AE index was $500-1100 \mathrm{nT}$. Intense chorus emissions were generated on the night side (Santolík et al., 2003a). The Cluster spacecraft were near their perigee at mutual separations of a few hundreds of km.

In this paper we show results of an improved analysis method and compare our results to those presented by Trakhtengerts et al. (2007). We analyze properties of chorus wave packets as a function of the position inside the generation region relative to the direction of the Poynting flux.

\section{Analysis of chorus wave packets}

Figure 1 shows an example of chorus measurements on 18 April 2002. Four panels respectively show frequency-time power spectrograms obtained from electric field measured on board the four Cluster spacecraft. This example represents $6 \mathrm{~s}$ of data from 08:46:04 UT. The electromagnetic planarity parameter (not shown) obtained from singular value decomposition of the electromagnetic spectral matrix (Santolík et al., 2003b) indicates that these observations were made inside the chorus source region (Santolík et al., 2004, 2005).
Using these high-resolution spectrograms we have visually determined the minimum and maximum time $t_{1}, t_{2}$ and the minimum and maximum frequency $f_{1}, f_{2}$ of each chorus wave packet. We have concentrated our analysis on the lower band of chorus below one half of the local electron cyclotron frequency $\left(f_{\mathrm{c} e}\right)$, where distinct chorus wave packets are often seen on the spectrograms (see Fig. 1). A digitization routine has been added to the PRASSADCO software (Santolík, 2003) and used to record these data. An example of visual determination of parameters of a chorus wave packet is shown on the bottom panel of Fig. 1 for Cluster 4. The red arrow indicates the starting point $\left[t_{1}, f_{1}\right]$ and the blue arrow shows the final point $\left[t_{2}, f_{2}\right]$ of a selected chorus wave packet.

Similar data have been recorded throughout the entire chorus source region within a few thousands of $\mathrm{km}$ around the geomagnetic equator. The extent of the source region was determined using the electromagnetic planarity as described by Santolík et al. (2004, 2005). Using the data of the four Cluster spacecraft this procedure yields a large set of coupled values $\left[t_{1}, f_{1}\right]$ and $\left[t_{2}, f_{2}\right]$. For example, from observations of Cluster 1 we have recognized 1140 wave packets. Accumulated data set from the four Cluster spacecraft contains 


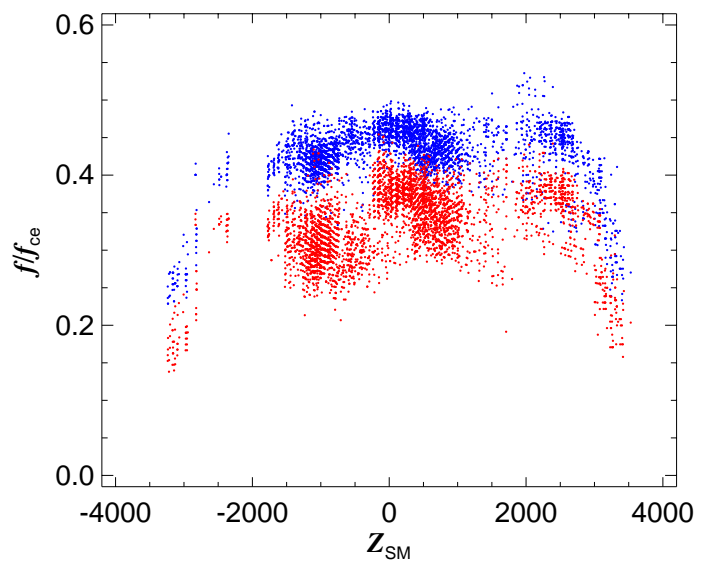

Fig. 2. Lower frequencies $f_{1}$ (red points) and upper frequencies $f_{2}$ (blue points) of each analyzed chorus wave packet normalized to local electron cyclotron frequency $f_{\text {ce }}$ as a function of the $Z_{\mathrm{S} M}$ coordinate for the 4682 analyzed wave packets recorded by the four Cluster spacecraft in the chorus source region.

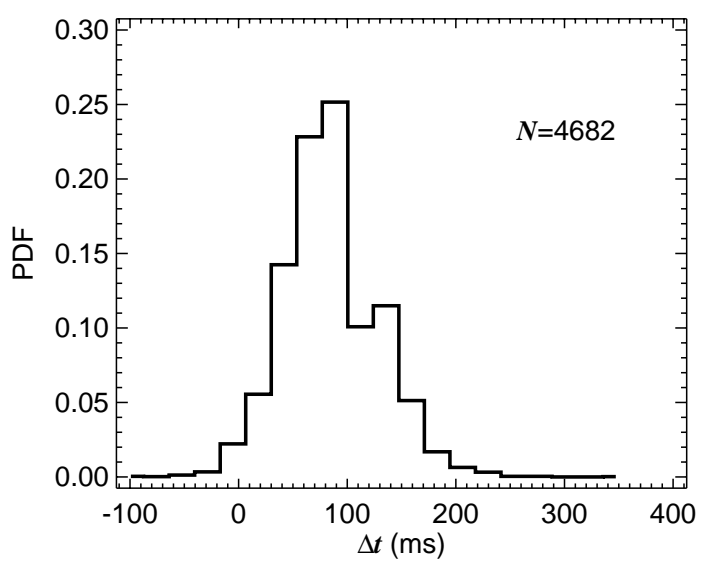

Fig. 3. An estimate of the probability density function of the duration of chorus wave packets. The same data set as in Fig. 2 has been used.

4682 wave packets. The frequencies $f_{1}$ (red points) $f_{2}$ (blue points) of each analyzed chorus wave packet from this data set have been normalized to the local electron cyclotron frequency $f_{\mathrm{c} e}$ and plotted as a function of the $Z_{\mathrm{S} M}$ coordinate in Fig. 2.

Using this data set, we have calculated the duration $\Delta t=t_{2}-t_{1}$ and the frequency bandwidth $\Delta f=f_{2}-f_{1}$ of every wave packet. Here, we have defined $f_{1}<f_{2}$, which means that a positive $\Delta t$ corresponds to a wave packet whose frequency increases (a riser) and a negative $\Delta t$ corresponds to a wave packet decreasing in frequency (a faller). Figure 3 shows a histogram which represents an estimate of the probability density function (PDF) of the obtained values of $\Delta t$. We can see that the majority of observed wave packets are risers whose duration ranges from a few milliseconds

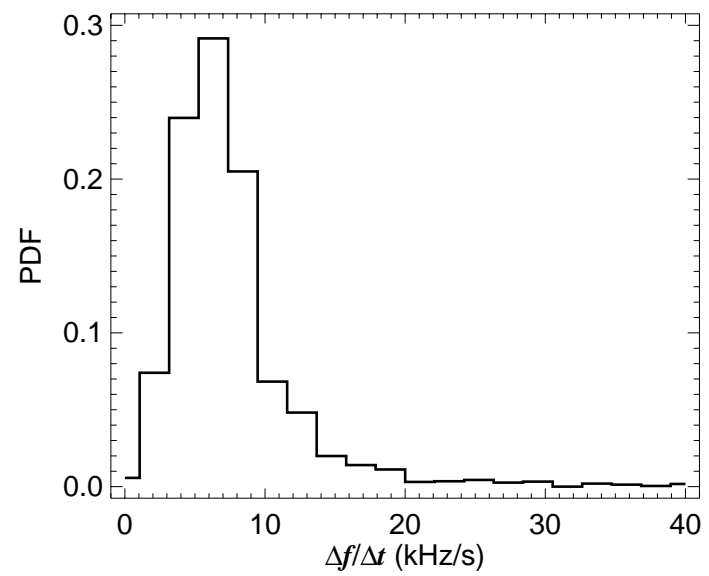

Fig. 4. An estimate of the probability density function of the sweep rate of chorus wave packets. The same data set as in Fig. 2 has been used.

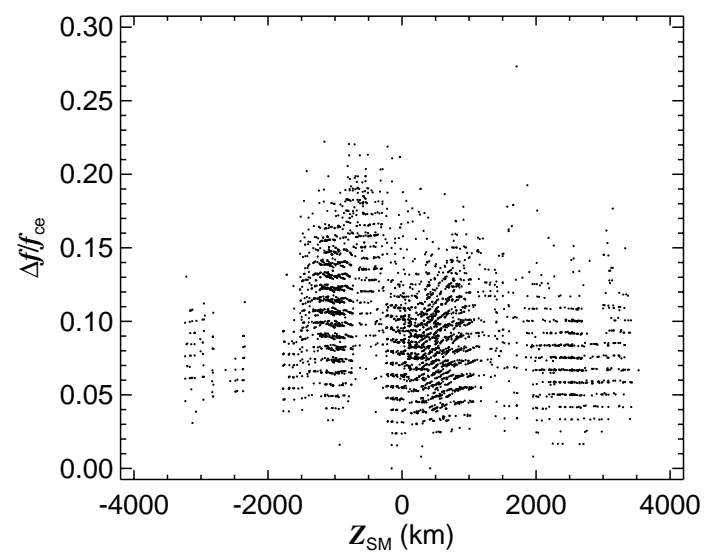

Fig. 5. Normalized frequency bandwidth $\Delta f / f_{\mathrm{c} e}$ as a function of the $Z_{\mathrm{S} M}$ coordinate for the 4682 analyzed wave packets recorded by the four Cluster spacecraft in the chorus source region.

to $200 \mathrm{~ms}$, with a maximum occurrence around $90 \mathrm{~ms}$. The corresponding histogram of the frequency sweep rate $\Delta f / \Delta t$ is presented in Fig. 4, giving the most frequent values of 6 $7 \mathrm{kHz} / \mathrm{s}$ and a median value of $7.6 \mathrm{kHz} / \mathrm{s}$. Figure 5 shows a scatter-plot of the normalized frequency bandwidth $\Delta f / f_{\mathrm{ce}}$ as a function of the $Z_{\mathrm{S} M}$ coordinate (perpendicular to the magnetic equator using the dipole model). Note that the horizontally oriented stripes on the plot are an artifact of a finite number of frequency bins used in the digitization procedure. The bandwidth of the majority of observed chorus wave packets varies from $0.03 f_{\mathrm{ce}}$ to $0.2 f_{\mathrm{c} e}$, with a maximum occurrence in the range $0.08-0.12 f_{\mathrm{ce}}$. The distribution seems to be shifted to slightly lower observed bandwidths very close to the dipole magnetic equator (at $Z_{\mathrm{S} M}=0$ ). 


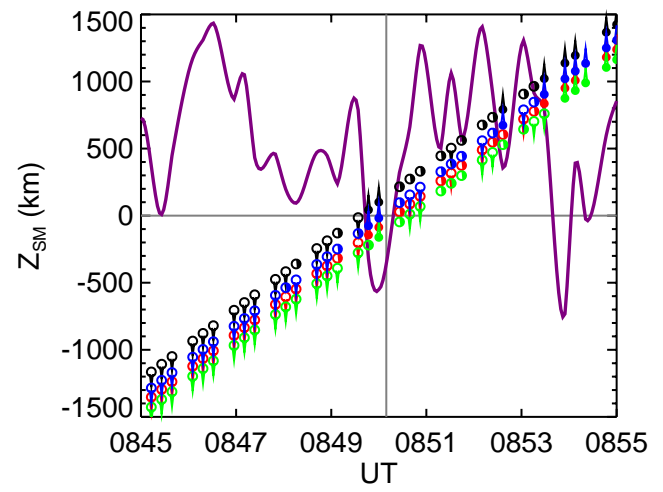

Fig. 6. Symbols represent variations of the sign of the parallel component of the Poynting flux of chorus along the orbit of the four Cluster spacecraft (black-C1, red-C2, green-C3, blue-C4) empty symbols with downward arrows mean predominant Southward propagation whereas solid symbols with upward arrows are used when chorus mainly propagates to the North. The half-solid symbols without arrow show data points where the sign of the parallel component of the Poynting flux was uncertain. Each symbol at the same time represents the $Z_{\mathrm{S} M}$ coordinate of the corresponding spacecraft as a function of time. The horizontal solid line shows the position of the magnetic equator; the vertical solid line shows the time when the center of mass of the four spacecraft crosses the equator. The purple line is the estimated central position of the chorus source region (Santolík et al., 2005).

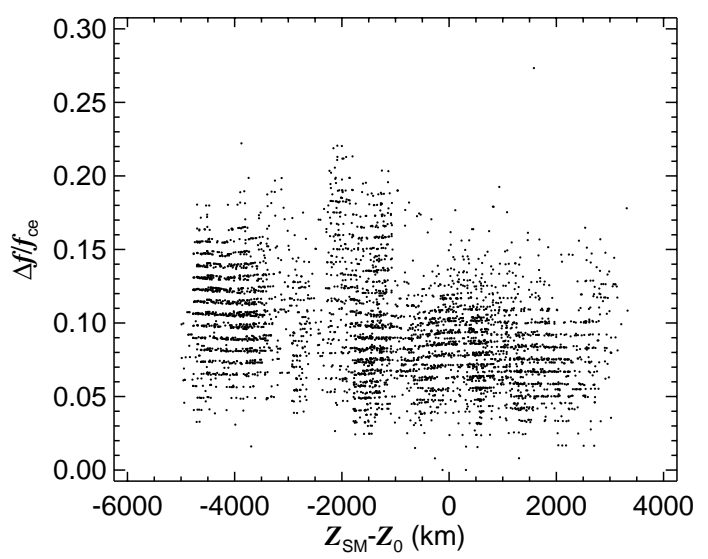

Fig. 7. The same as in Fig. 5 but as a function of the distance of the point of observation from the central position of the chorus source region measured along the $Z_{\mathrm{S} M}$ coordinate.

\section{Relation of frequency band of chorus wave packets to the central position of the source region}

Poynting flux measurements of have shown that chorus is generated close to the dipole magnetic equatorial plane and that it propagates outwards from the source region (LeDocq et al., 1998; Parrot et al., 2003; Santolík et al., 2003a). This means that the most important component of the Poynting flux is antiparallel to the terrestrial magnetic field at nega-

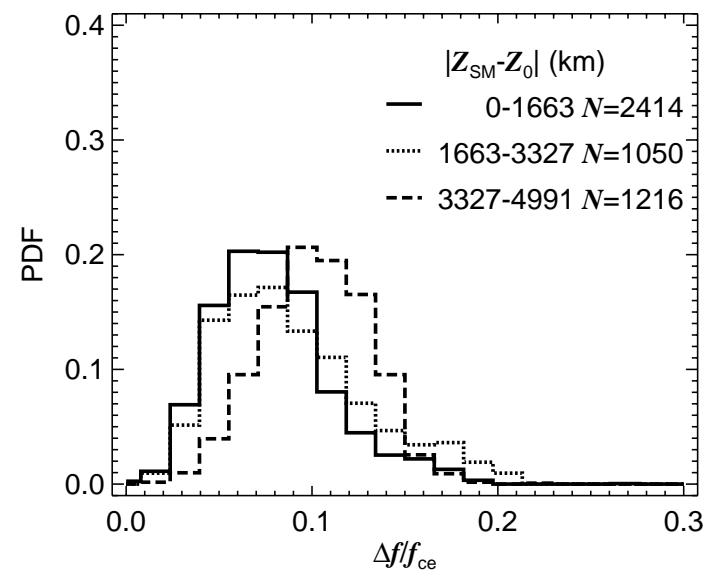

Fig. 8. Histograms of the probability density function of the normalized frequency bandwidth estimated for three intervals of the distance from the central position of the chorus source region measured along the $Z_{\mathrm{S} M}$ coordinate. Histograms plotted by solid and dotted lines are calculated using data observed closer to the central position, dashed line means observations closer to the outer boundaries of the source region. The numbers $N$ of individual wave packets falling into the three intervals are, respectively, given on the right-hand side.

tive magnetic latitudes and parallel to it at positive latitudes. However, detailed multi-component wave measurements of the STAFF instruments (Cornilleau-Wehrlin et al., 2003) onboard the four Cluster spacecraft revealed that the direction of the Poynting flux does not always switch from parallel to antiparallel at once at the dipole equator. Instead, a spacecraft which passes through the equatorial plane observes that, close to the equator, the parallel component of the Poynting flux fluctuates between significantly negative and significantly positive values (Santolík et al., 2003a).

Importantly enough, these fluctuations, as seen by the four closely separated Cluster spacecraft, correspond to the motion of the position where the parallel component of the Poynting flux reverses its sign (Santolík et al., 2005). The temporal evolution of this position has been estimated by a multi-point technique based on the normalized parallel component of the Poynting flux, averaged in the frequency interval of the lower band of chorus (below $4 \mathrm{kHz}$ ) and spatially interpolated or extrapolated to its zero points (Santolík et al., 2005). Since each data point always comprises many separate wave packets of chorus, this estimate gives an average global position of the center of the chorus source region. Kozelov et al. (2008) have shown that this position most probably follows fluctuations of the minimum of the geomagnetic field strength (the "true" geomagnetic equator) along a given field line.

Figure 6 shows the central position of the chorus source region resulting from this method as a dotted line. The position is defined by its $Z_{\mathrm{S} M}$ coordinate as a function of time. The $Z_{\mathrm{S} M}$ coordinates of the four spacecraft are shown in Fig. 6 
(a)

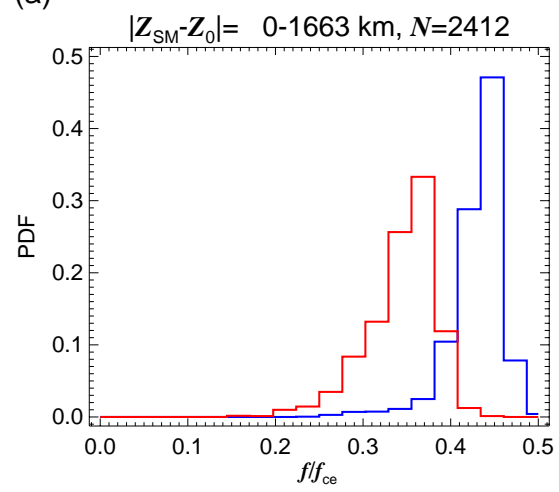

(b)

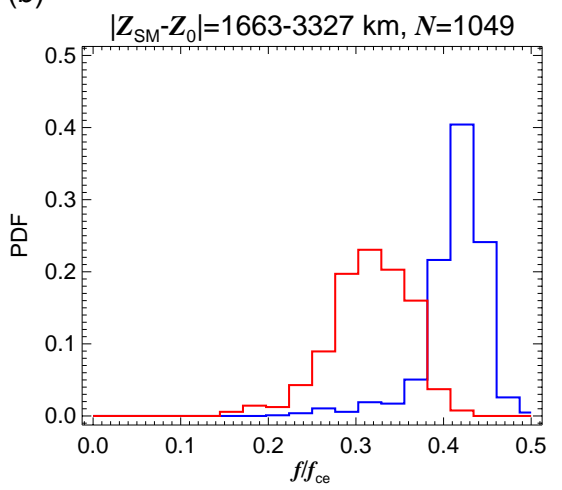

(c)

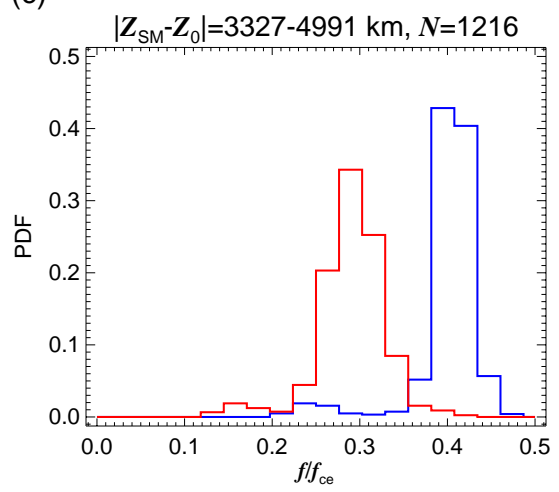

Fig. 9. Estimates of the probability density functions of lower frequencies $f_{1}$ (red lines) and upper frequencies $f_{2}$ (blue lines) of individual chorus wave packets. The three panels (a), (b), and (c) show, respectively, the results for three different intervals of distances $Z_{\mathrm{S} M}-Z_{0}$ from the central position of the source region.

as functions of time by circular symbols. At the same time these symbols reflect the variations of the sign of the parallel component of the Poynting flux along the orbits of the spacecraft. The estimated central position of the source separates regions with predominant Northward (solid symbols with upward arrows) and Southward (empty symbols with downward arrows) propagation.

The estimated central position of the chorus source region fluctuates within hundreds to thousands of $\mathrm{km}$ off the dipole geomagnetic equator. The amplitude of these fluctuations is comparable to the extent of the source region. It is therefore natural to relate the properties of chorus wave packets to the distance from this central position instead of using the distance from the dipole geomagnetic equator. Figure 7 shows again a scatterplot of the normalized frequency bandwidth of chorus wave packets but this time as a function of the separation of the point of observation from the central position of the source region measured along the $Z_{\mathrm{S} M}$ coordinate, $Z_{\mathrm{S} M}-Z_{0}$. Here, $Z_{\mathrm{S} M}=Z_{0}$ means the position of the estimated central position of the chorus source. The results indicate a tendency of slightly lower bandwidths for chorus wave packets observed close to the central position of the source.

In Fig. 8 we represent the same data in the form of estimates of the probability density function of the normalized frequency bandwidth. The three histograms are, respectively, constructed for three different intervals of distances $Z_{\mathrm{S} M}-Z_{0}$ from the central position of the source region. Close to the central position, the most probable bandwidths are around 0.07-0.08 $f_{\mathrm{ce}}$. Further away, at distances above $\approx 3000 \mathrm{~km}$, the most probable bandwidths move upward to $\approx 0.1 f_{\mathrm{ce}}$ and the entire peak of probability density shifts toward larger bandwidths.

These results are consistent with conclusions of Trakhtengerts et al. (2007) who expect lower bandwidth of chorus wave packets close to the center of the source region. They further indicate that lower frequencies are absent from cho- rus wave packets close to the center of the source region. Figure 9 shows that this is indeed confirmed by our improved analysis. The lower frequencies $f_{1}$ and the upper frequencies $f_{2}$ of the individual chorus wave packets are used to calculate separate estimates of the probability density functions. The results are compared for three intervals of distances from the central position of the source region. We can notice that, with increasing distance from the central position, the peak of the probability density of the upper frequencies slightly shifts downward from $f_{2}=0.45 f_{\mathrm{ce}}$ to $f_{2}=0.4 f_{\mathrm{ce}}$. More importantly, the peak of the probability density of the lower frequencies moves down from $f_{1}=0.38 f_{\mathrm{c} e}$ to $f_{1}=0.29 f_{\mathrm{ce}}$. This indicates that close to the central position of the source region the observed chorus wave packets are more confined to higher frequencies.

\section{Conclusions}

We have analyzed a chorus event recorded by the four Cluster spacecraft on the night side during geomagnetically disturbed conditions.

Inside the chorus source region, identified using the electromagnetic planarity estimator, we have analyzed lower and upper frequencies for $\sim 4700$ individual chorus wave packets at typical time scales of $100 \mathrm{~ms}$ and sweep rates of $8 \mathrm{kHz} / \mathrm{s}$. We have related these results to the separation from the central position of the chorus source estimated from the Poynting flux measurement.

The typical frequency bandwidths of the individual chorus wave packets are of the order of $10 \%$ of the electron cyclotron frequency and tend to be larger close to the boundaries of the source region located a few thousands of $\mathrm{km}$ along the magnetic field line from its central position. Close to the central position the chorus wave packets tend to be more confined to higher frequencies. These results are in agreement with a hypothesis on frequencies of individual 
chorus wave packets based on the BWO theory (Trakhtengerts et al., 2007).

Acknowledgements. This paper is dedicated to the memory of our colleague V. Y. Trakhtengerts, recently deceased. We acknowledge the access to the spin-resolution data of the FGM magnetic field experiment (provided by A. Balogh and E. Lucek). We thank R. Huff, J. Dowell, J. Seeberger and other colleagues from the University of Iowa for the calibration and preprocessing of the WBD measurements. This work was supported by INTAS (grant 0351-4132). A. G. Demekhov, E. E. Titova, and B. V. Kozelov acknowledge a support from the Russian Foundation for Basic Research (grants 05-02-16459 and 04-05-64955) and from the Program for Basic Research "Plasma Processes in the Solar System" of the Russian Academy of Sciences. The WBD measurements were supported by the NASA Goddard Space Flight Center under Grants No. NNX07AI24G and NNG04GB98G. O. Santolik and E. Macusova acknowledge additional support from the NSF award No. 0307319/ME 842 and GAAV grant IAA 301120601.

Topical Editor I. A. Daglis thanks M. L. Kaiser and another anonymous referee for their help in evaluating this paper.

\section{References}

Balogh, A., Carr, C. M., Acuña, M. H., Dunlop, M. W., Beek, T. J., Brown, P., Fornaçon, K.-H., Georgescu, E., Glassmeier, K.-H., Harris, J., Musmann, G., Oddy, T., and Schwingenschuh, K.: The Cluster Magnetic Field Investigation: overview of in-flight performance and initial results, Ann. Geophys., 19, 1207-1217, 2001 , http://www.ann-geophys.net/19/1207/2001/.

Cornilleau-Wehrlin, N., Chanteur, G., Perraut, S., Rezeau, L., Robert, P., Roux, A., Villedary, C. d., Canu, P., Maksimovic, M., Conchy, Y. d., Hubert, D., Lacombe, C., Lefeuvre, F., Parrot, M., Pincon, J.-L., Decrau, P., Harvey, C. C., Louarn, P., Santolik, O., Alleyne, H. S. C., Roth, M., et al.: First results obtained by the Cluster STAFF experiment, Ann. Geophys., 21, 437-456, 2003, http://www.ann-geophys.net/21/437/2003/.

Gurnett, D. A., Huff, R. L., and Kirchner, D. L.: The Wide-Band Plasma Wave Investigation, Space Sci. Rev., 79, 195-208, 1997.

Gurnett, D. A., Huff, R. L., Pickett, J. S., Persoon, A. M., Mutel, R. L., Christopher, I. W., Kletzing, C. A., Inan, U. S., Martin, W. L., Bougeret, J.-L., Alleyne, H. S. C., and Yearby, K. H.: First results from the Cluster wideband plasma wave investigation, Ann. Geophys., 19, 1259-1272, 2001, http://www.ann-geophys.net/19/1259/2001/.

Helliwell, R. A.: A theory of discrete emissions from the magnetosphere, J. Geophys. Res., 72, 4773-4790, 1967.

Kozelov, B. V., Demekhov, A. G., Titova, E. E., Trakhtengerts, V. Y., Santolik, O., Macusova, E., Gurnett, D., and Pickett, J.: Variations in the chorus source location deduced from fluctuations of the ambient magnetic field: comparison of CLUSTER data and the backward-wave oscillator model, J. Geophys. Res., in press, doi:10.1029/2007JA012886, 2008.
LeDocq, M. J., Gurnett, D. A., and Hospodarsky, G. B.: Chorus source locations from VLF Poynting flux measurements with the Polar spacecraft, Geophys. Res. Lett., 25, 4063-4066, 1998.

Nunn, D., Omura, Y., Matsumoto, H., Nagano, I., and Yagitani, S.: The numerical simulation of VLF chorus and discrete emissions observed on the Geotail satellite using a Vlasov code, J. Geophys. Res., 102, 27 083-27 097, 1997.

Omura, Y., Nunn, D., Matsumoto, H., and Rycroft, M. J.: A review of observational, theoretical and numerical studies of VLF triggered emissions, J. Atmos. Terr. Phys., 53, 351-368, 1991.

Parrot, M., Santolík, O., Cornilleau-Wehrlin, N., Maksimovic, M., and Harvey, C.: Source location of chorus emissions observed by Cluster, Ann. Geophys., 21, 473-480, 2003, http://www.ann-geophys.net/21/473/2003/.

Santolík, O.: Propagation Analysis of STAFF-SA Data with Coherency Tests (A User's Guide to PRASSADCO), Tech. rep., Lab. Phys. Chimie Environ./CNRS, Orléans, France, 2003.

Santolík, O., Gurnett, D. A., Pickett, J. S., Parrot, M., and Cornilleau-Wehrlin, N.: Spatio-temporal structure of stormtime chorus, J. Geophys. Res., 108, 1278, doi:10.1029/ 2002JA009791, 2003a.

Santolík, O., Parrot, M., and Lefeuvre, F.: Singular value decomposition methods for wave propagation analysis, Radio Sci., 38, 1010, doi:10.1029/2000RS002523, 2003b.

Santolík, O., Gurnett, D. A., Pickett, J. S., Parrot, M., and Cornilleau-Wehrlin, N.: A microscopic and nanoscopic view of storm-time chorus on 31 March 2001, Geophys. Res. Lett., 31, L02801, doi:10.1029/2003GL018757, 2004.

Santolík, O., Gurnett, D. A., Pickett, J. S., Parrot, M., and Cornilleau-Wehrlin, N.: Central position of the source region of storm-time chorus, Planet. Space Sci., 53, 299-305, 2005.

Sazhin, S. S. and Hayakawa, M.: Magnetospheric chorus emissions: A review, Planet. Space Sci., 40, 681-697, 1992.

Trakhtengerts, V.: Magnetosphere cyclotron maser: backward wave oscillator generation regime, J. Geophys. Res., 100, $17205-$ $17210,1995$.

Trakhtengerts, V.: A generation mechanism for chorus emission, Ann. Geophys., 17, 95-100, 1999, http://www.ann-geophys.net/17/95/1999/.

Trakhtengerts, V. and Rycroft, M.: Whistler electron interactions in the magnetosphere: new results and novel approaches, J. Atmos. Sol.-Terr. Physics, 62, 1719-1733, 2000.

Trakhtengerts, V. Y., Demekhov, A. G., Titova, E. E., Kozelov, B. V., Santolik, O., Gurnett, D., and Parrot, M.: Interpretation of Cluster data on chorus emissions using the backward wave oscillator model, Phys. Plasmas, 11, 1345-1351, 2004.

Trakhtengerts, V. Y., Demekhov, A. G., Titova, E. E., Kozelov, B. V., Santolik, O., Macusova, E., Gurnett, D. A., Pickett, J. S., Rycroft, M. J., and Nunn, D.: Formation of VLF chorus frequency spectrum: Cluster data and comparison with the backward wave oscillator model, Geophys. Res. Lett., 34, L02104, doi:10.1029/2006GL027953, 2007.

Tsurutani, B. T. and Smith, E. J.: Postmidnight chorus: a substorm phenomenon, J. Geophys. Res., 79, 118-127, 1974. 